\title{
Methodology of Low Cost Rapid Manufacturing of Anatomical Models with Material Imitation of Soft Tissues
}

\author{
Magdalena Żukowska ${ }^{1 *}$, Filip Górski', Radosław Wichniarek', Wiesław Kuczko \\ 1 Chair of Management and Production Engineering, Poznan University of Technology, ul. Piotrowo 3, \\ 61-138 Poznan, Poland \\ * Corresponding author's e-mail: magdalena.k.zukowska@doctorate.put.poznan.pl
}

\begin{abstract}
The pre-operative preparation of a surgeon is increasingly often extended to individual patient's anatomical models depicting the affected organ and the existing lesion in relation to the surrounding structures (e.g. blood vessels). Among the models, there are models of the skeletal system, in which the FFF technology is used. The models of parenchymal organs are more difficult to reproduce due to the material limitations in the FFF technology, which is why alternative technologies such as PolyJet and SLA are used. Due to the high price of models, they are employed less frequently, especially in the Polish hospitals. The research presented in the article deals with the modification of the existing methodology of rapid pre-operative models production with the use of various low-budget rapid manufacturing techniques, which use materials of low hardness, similar to the human soft tissues. The aim was to create a methodology and determine the parameters of materials that allow for the cheap production of functional pre-operational aids.
\end{abstract}

Keywords: 3D Printing, Pre-operative support, Biomedical Engineering

\section{INTRODUCTION}

The modern development of additive manufacturing technologies (popularly known as 3D printing) contributes to an increased use of technology in a growing group of services, industry or transport. In addition, increased use is also observed in medicine in various areas. Increasingly intensive research on bioprinting is a response to the high demand for organs in the field of transplantology and is possible due to the continuous development of additive manufacturing technology. The research on this topic concerns both the external structures of the human body, such as ears, skin or nose [11] and internal organs: liver, heart, kidneys $[3,7]$. The implantology is another area of medicine that uses the 3D printing technology. It is possible to obtain highly complex shapes that reproduce the exact anatomy of bone defects and allow for the rapid manufacturing of individual implants and endoprostheses. In the literature, there are examples of rib and sternum implants adapted to the patient's bone system, hip joint endoprostheses or individual jaw implants [1]. The applied technology for implants is DMLS (Direct Metal Laser Sintering) and it uses titanium powders that are biocompatible and bioactive as a building material (they facilitate the work of bone cells in the reconstruction process). A similar application for the FFF / FDM (Fused Deposition Modeling) technology is used in the rehabilitation process. Durable parts for medical lifts can also be manufactured additively [5]. Plasticized plastics such as ABS, PLA or HIPS may produce external dentures and orthoses dedicated for a specific patient $[8,10]$. In the case of the anatomical models created based on individual medical imaging of the patient (CT / MRI), various rapid manufacturing technologies are used, depending on the requirements of the medical models and the purpose in which they were created. The models for pre-operative support may serve as a demonstration model, helping the patient and his family understand the existing problem [9]. It can 
be a model used in the pre-operative preparation when planning the course of surgery. Then, the doctor becomes acquainted with the pathological lesion, healthy structures and relations between them [9]. In this way, the time of surgery is shortened $[1,2]$, determines how to reach the operating area, increases the confidence of the doctor and the patient's safety [9]. In addition, the model can provide educational help for medical students, trainees and residents at every stage. Moreover, the models can be used to perform a simulated operation and at this level, the model must consist of different materials with different mechanical properties [6]. The task of the model is to reliably reflect the individual situation of the patient. Imitation of individual structures, organs, blood vessels and pathological changes is required. At this stage, it is important to choose the right technology for rapid manufacturing or the use of several technologies. Depending on the guidelines of the client's model and financial capabilities, it may be necessary to modify the standard methodology used to manufacture the anatomical model.

The aim of the research was, based on the information obtained so far, to define the methodology of producing multi-material personalized anatomical models that can be used to perform simulated surgery. The basis for the correct determination of the process was the selection of the rapid manufacturing methods and materials used in the process. The conditions set by the authors of the study were first and foremost the low price of receiving the finished product while maintaining the high quality of the model and the reliable imitation of the kidney soft tissues.

\section{MATERIALS AND METHODS}

\section{Case and problem definition}

The manufacturing of multi-material models can take place using various technologies. Some of the available rapid manufacturing methods allow for the simultaneous production of specific elements in a model from different materials and everything takes place in a single process. Commercial dual-head devices are available for the FDM method, which allow color mixing, but it is not possible to produce the models with very different materials for mechanical properties. The possibility of mixing materials is also available in the MJM (Multi Jet Modeling) as well as the more developed PolyJet and Triple Jet methods. By applying the resin and its derivatives in the form of drops and then curing with UV light, they enable the creation of composite materials in a precisely defined digital way. These are currently the most suitable technologies for creating a functional anatomical model of organs made of soft tissues in one process. The model's functionality in this case refers to the possibility of performing a simulated operation.

The closure of the entire manufacturing process in one cycle is of special importance due to the time that is particularly important in medical situations [1]. However, with the use of PolyJest technology, there are large costs of the model, which in the case of not very complicated operations (e.g. educational) or medium complex (determining the exact location of the change and taking a decision to remove the organ or change

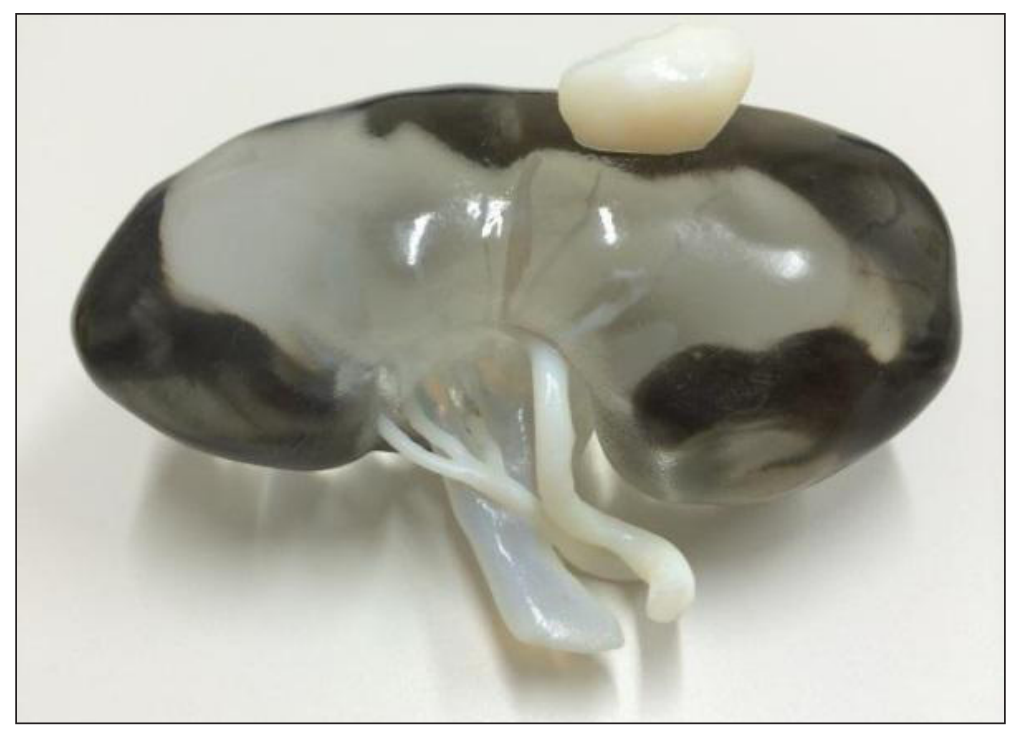

Fig. 1. An example of a multi-material model of the kidney [2] 
the operation area), may be disproportionately high relative to the cost of the entire operation. In the case of the middle-income countries, there are no chances that such models would be employed everyday in hospitals and regularly implemented at various stages of education and pre-operational support. It is an economic factor that can negate the purpose of using such models in general.

Therefore, it was necessary to modify the approach to the topic and introduce a multi-stage modeling using a variety of incremental production methods.

The methodology for producing an individual anatomical model is shown in Fig. 2. An individual anatomical model is obtained based on the patient's medical imaging, for example, computerized tomography or magnetic resonance. The medical data in the form of a DICOM file (Digital Imaging and Communications in Medicine) is used to perform segmentation in MIMICS or InVesalius medical software. The obtained digital 3D model of the organ, lesion and other structures has been subjected to digital post processing and removal of errors occurring in the triangle mesh. The finished model in the STL format is transferred to the software dedicated to the device for rapid manufacturing. The produced model is subjected to final post processing [4].

\section{Materials}

In order to modify the existing methodology, it was necessary to determine which of the available rapid manufacturing methods would allow a low-budget creation of a functional model. From the available methods, it was decided to designate three of them: FDM, SLA / DLP and Vacuum Casting. The process of selecting the method was closely related to the choice of material, its price and selected mechanical parameters. The materials considered are presented in Tab. 1 .

The most important requirement of the materials was hardness, which was to be similar to the hardness of soft human tissue. Elasticity, susceptibility to plastic deformation (due to the process of surgical suturing), price and transparency were equally important parameters. The prerequisite for setting these specific parameters was a medical consultation with a specialist physician who could identify them, based on his own experience and familiarization with the example model, along with performing a simulated operation.

\section{Test procedure for materials}

The selection of materials was based on the subjective evaluation of the animal model (pig kidney), experience of communing with the human organ (medical consultation) and knowledge gained at the stage of medical studies (questionnaire studies).

The first step was a medical consultation on the aforementioned exemplary model, made of silicone with a hardness of 45 Shore A. The surgical suturing process and empirical assessment enabled to determine that the material is too hard and too stiff. This led directly to breaking the seams and tearing the material. Therefore, this hardness was defined as the upper limit of the range for further sought-after materials. The lower limit was the value of 5 Shore A. During the selection of materials for the aforementioned methods, the materials with such a low hardness were not always available. Therefore, in these cases, the material with the lowest possible hardness (along with the remaining parameters) or material for biomedical applications was selected instead.

After the selection of materials (Table 1), one of the technologies was selected to further narrow the group of materials. The aim was to find the material nearest the soft tissue of the kidney cortex. Considering the mentioned determinants, it was decided that the materials selected for the VC method (Vacuum Casting) have the best hardness, flexibility, transparency, susceptibility to sewing and the price translating into time.

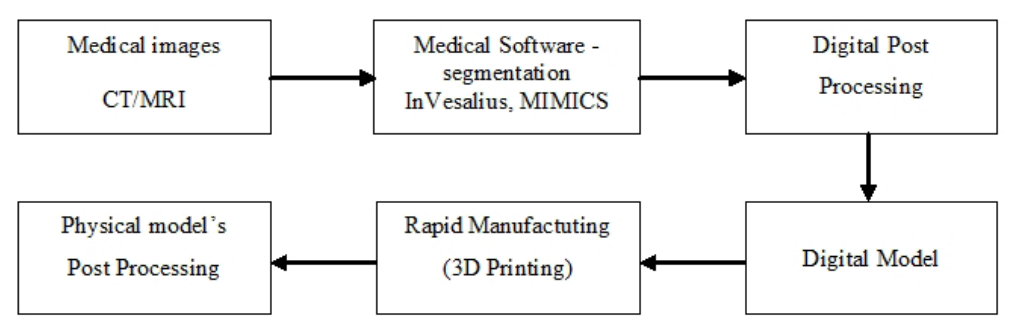

Fig. 2. Methodology of anatomical model manufacturing based on medical imaging [based on: 4] 
Table 1. List of selected materials with a specific hardness ( $\sim 5$ Shore A - 45Shore A / biomedical) [based on material technical sheets]

\begin{tabular}{|c|l|l|c|l|}
\hline Method & \multicolumn{1}{|c|}{ Type of material } & \multicolumn{1}{|c|}{ Name } & Hardness [Shore A] & \multicolumn{1}{c|}{ Color } \\
\hline VC & Silicone & ZA 8 LT & 8 & Transparent \\
\hline VC & Silicone & ZA 22 Mould & 21 & White \\
\hline VC & Silicone & Dragon Skin 10 Very Fast & 10 & Semitransparent \\
\hline VC & Silicone & Sorta Clear 12 & 12 & Transparent \\
\hline VC & Silicone & Sorta Clear 37 & 37 & Transparent \\
\hline VC & Silicone & Encapso K & 20 & Transparent \\
\hline VC & Silicone & Body Double Standard & 25 & Blue \\
\hline VC & Resin & GM-900-1 & 13 & Yellow \\
\hline VC & Resin & GM 956-45 & 45 & Amber \\
\hline DLP & Resin & Resin Flexible & -- & Transparent \\
\hline DLP & Resin & SuperFine & -- & Purple \\
\hline SLA & Resin & SuperFine Clear & -- & Transparent \\
\hline FDM & PCL & eSun eMate & 40 & Red \\
\hline FDM & Experimental & Lay Fomm 40 & 60 & Beige \\
\hline FDM & Experimental & Lay Fomm 60 & $20-30$ & Beige \\
\hline FDM & Experimental & GEL LAY & 85 & Semitransparent \\
\hline FDM & Modified TPE & Ninja Flex Water & & \\
\hline
\end{tabular}

The next step in narrowing the group of materials to accurately determine materials and mechanical properties was to refer to the animal pattern. For example, a pig kidney was used that is the closest to human. Two types of sewing were employed: sewing along the incision on the surface of the kidney cortex and removing two membranes not resulting from dissection. In this way, the subjective hardness and susceptibility to plastic deformation were assessed. There was also a significant behavior of the tissue - propagation of the material walls after cutting. Then, the abovementioned tests were reproduced analogously on the samples of silicone and resin materials.

The samples on which the sewing was made were divided into two groups:

- renal cortex samples: 55x55x25 mm,

- membrane after tumor removal: 50x14x3 mm.

Sewing of individual samples was performed with 3 types of surgical threads. Unworn threads of sizes $1-0$ and 3-0 were used as well as 3-0 absorbed threads. The samples were cut as in the case of surgery by means of a scalpel No. 11. The silicone samples subjected to sewing are shown in the pictures below (Figures 3, 4).

Silicone samples, due to the conflict with the silicone mold, could not be cast in traditional forms, which is why they were made for them using the FDM method (material: ABS), (Fig. 5). In the case of the resin models, the molds present at the state of the Rapid Manufacturing Laboratory of Poznan University of Technology were used (Fig. 5).
In order to supplement the subjective assessment of the authors, an identical sample for a larger number of samples and stitches was performed by a group of medical students (3rd Year). The participants of the study were asked to perform surgical suturing using the basic single node method, or another known one (Fig. 7). On each sample, the participants had to perform at least one stitch with each thread $(3-0,3-0,1-0)$, received one sample imitating the kidney cortex and the kidney membrane from each material. After the sewing process, each participant answered the questions related to the feelings about hardness, imitation of soft tissue, ease of sewing and general questions, having an educational character.

In order to summarize the answers in the survey, the materials were evaluated as follows (Table 2).

\section{DISCUSSION OF RESULTS}

The obtained answers of both authors and students allowed selecting the materials that are closest to the kidney tissue, enabling free cutting, manipulation and sewing of samples. The summary stage for the materials involved the observation of the changes taking place in the samples, such as: cracking of seams, propagation of seams, tearing of the material due to too high tension inside the seams and others. The observational data was recorded three times: immediately after surgical 

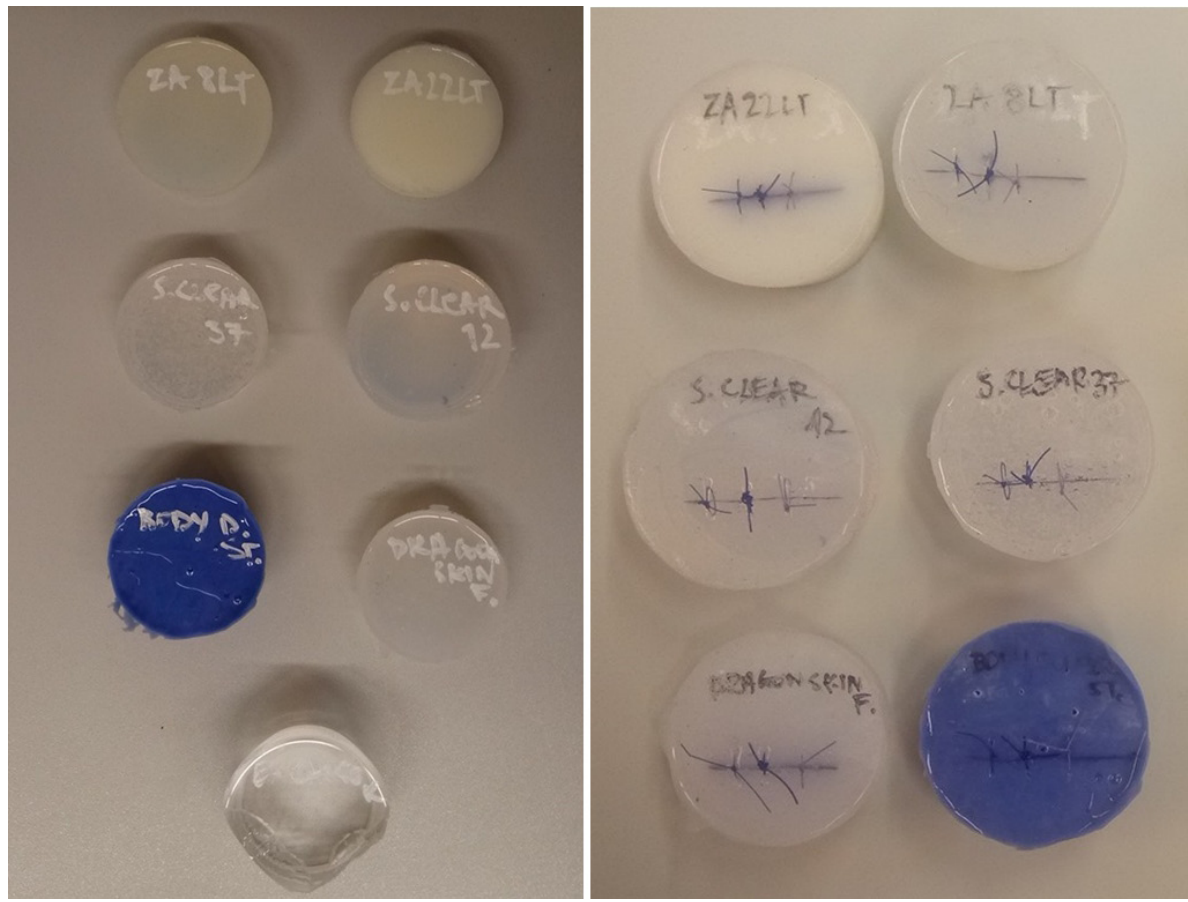

Fig. 3. Samples of materials before tests (left) and after suturing (right)

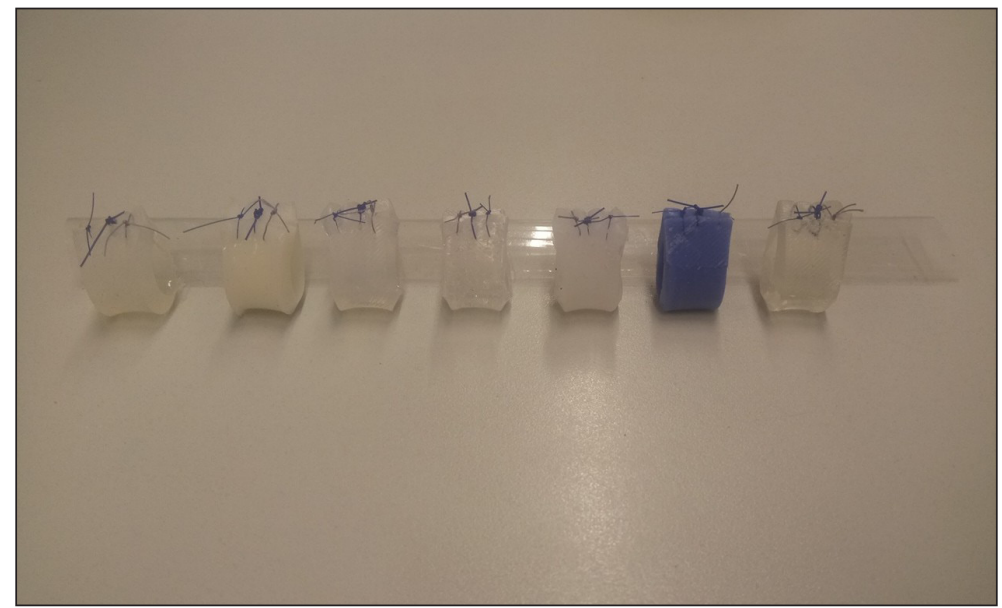

Fig. 4. Samples after suturing, process of connecting two edges create strain inside material and sutures
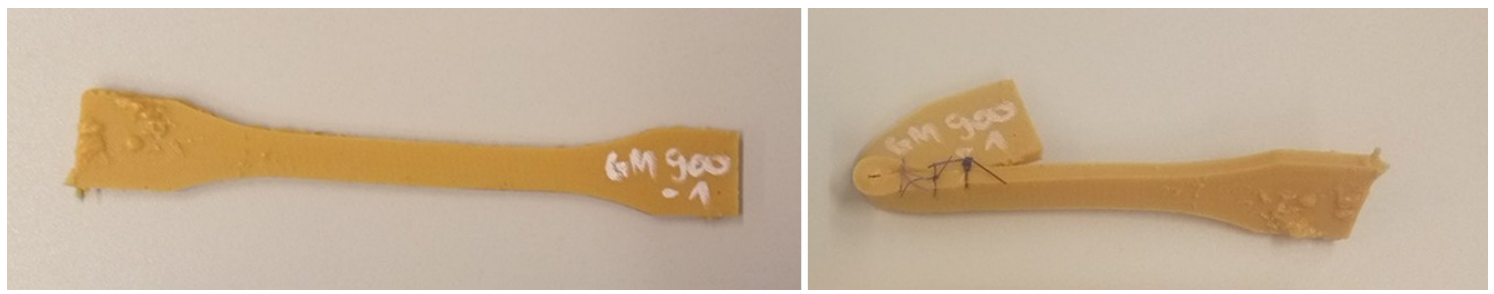

Fig. 5. Resin samples before (left) and after (right) suturing

suturing, two hours after the procedure and two weeks after the examination. The most significant changes were noted in the first and third intervals.

The changes for both types of samples were similar. The material disqualified at the beginning of the research, the Encapso K silicone, having
$100 \%$ transparency, but with high stiffness and brittleness, which prevented any operations related to sewing, cutting, etc. Material ZA 8 LT, with a hardness of 8 Shore A was too soft, which made sewing difficult and differed from the imitation of soft kidney tissue. In addition, in the 


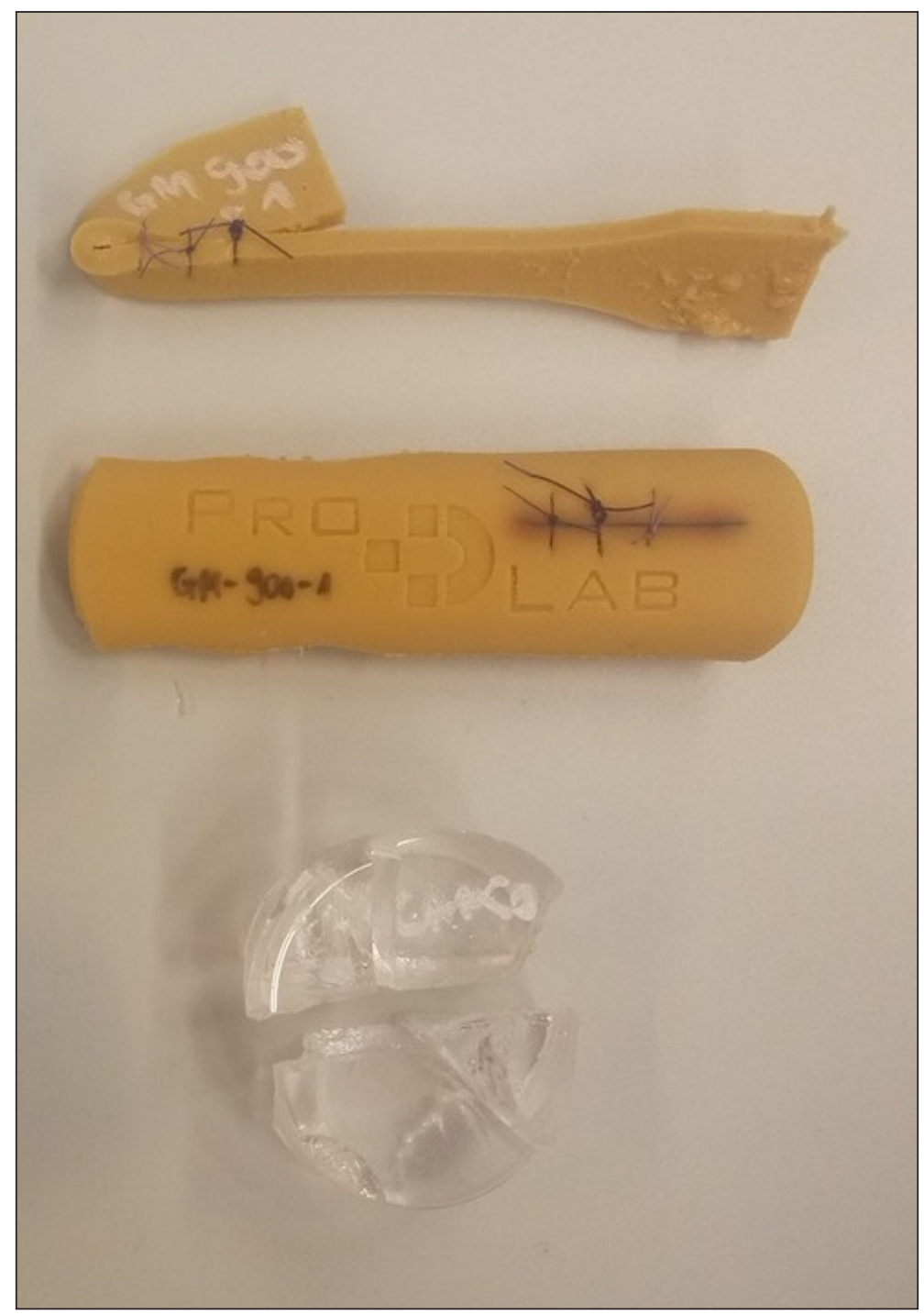

Fig. 6. Resin sample imitating renal cortex after suturing (top) and damaged sample of silicone Encapso K (down)

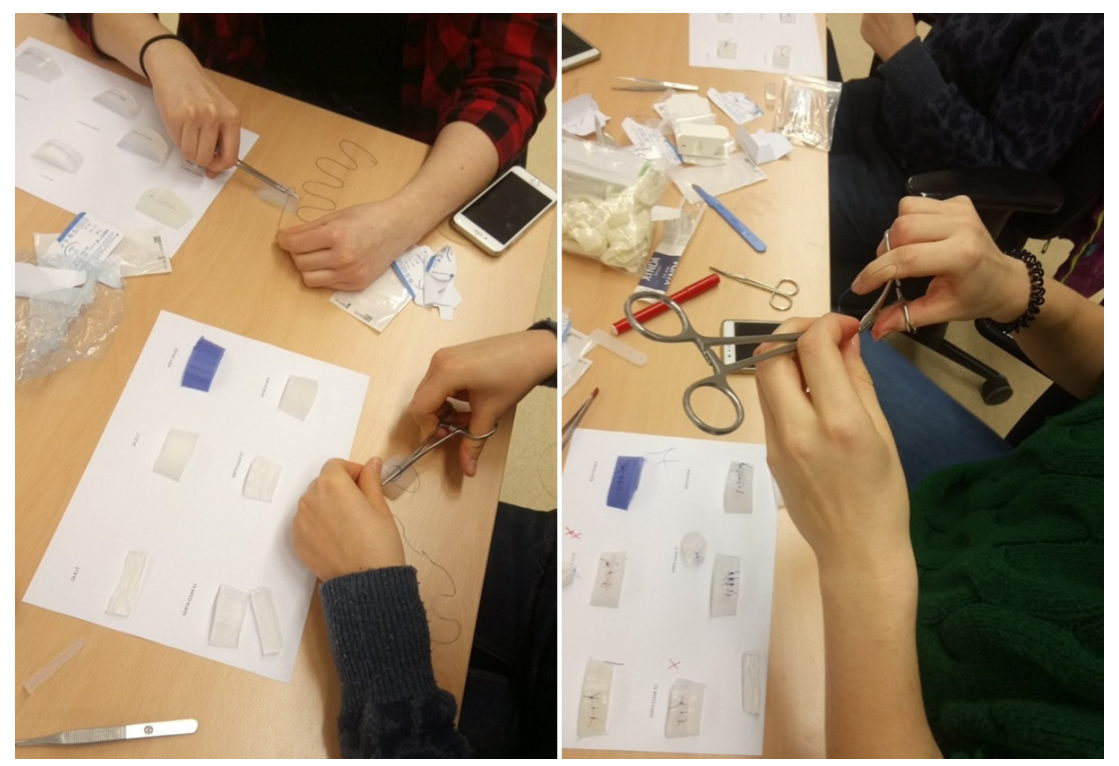

Fig. 7. The process of suturing samples by students 
Table 2 Collected answers to questions about feelings related to materials

\begin{tabular}{|c|c|c|c|c|c|c|}
\hline Question Material & Za 8 It & Za 22It & Body double & Sorta clear 12 & Sorta clear 37 & Dragon skin \\
\hline Easiness of cutting & 3 & 2.6 & 3 & 2.8 & 2.8 & 3.6 \\
\hline Easiness of sutituring & 3 & 3 & 3.2 & 2.8 & 3.4 & 3.6 \\
\hline Easiness of removing stitches & 2.8 & 3 & 3.2 & 2.6 & 3 & 4.2 \\
\hline Imitation of soft tissue & 2.8 & 3.4 & 2.4 & 2.2 & 2.6 & 3.8 \\
\hline Subjective evaluation of the material & 2.6 & 3.4 & 3.8 & 2.8 & 2.8 & 4.4 \\
\hline
\end{tabular}

case of ZA 8LT and Sorta Clear 12, the material was torn open by the stitches just after suturing and was still but slightly progressing. The cuts after two weeks were recorded in the materials Body Double and ZA 22 LT. For the samples made of GM 900-1 resin, problems dependent on the casting mold were noted. Casting of the material in the open mold produced by incremental methods had a negative effect on the hardening of the material, which was not noticed when using closed silicone molds. In the case of Sorta Clear 12 and 37, a degassing process is necessary due to the high gas content of the material and the formation of air bubbles. This process extends the time necessary to create models. In addition, the hardness of the models deviates from the assessment obtained on the animal model. The best answer among all tested materials was obtained for Dragon Skin 10 Very Fast. The material is susceptible to surgical suturing, easily subjected to deformation during sewing, and its hardness is similar to the kidney tissue. The defects in the material should be attributed to transparency, which is quite low. However, the model is easy to prepare, hardens quickly (30 minutes), does not require additional degassing and does not react with the mold produced by FDM from materials such as ABS. The table below shows the selected parameters for the material (Table 3).

The cost of the material is 53 USD for $0.91 \mathrm{~kg}$ of material (components A and B). The average kidney volume is about $0.2-0.3 \mathrm{~kg}$. The calculated the cost of material needed to create an anatomical model of the kidney is 11-17 USD. Positive indirect costs related to modification of the process of obtaining a ready model are necessary for the material costs - production costs of the mold (material, device work), and engineer's work (segmentation and mold design).

\section{Methodology}

Due to the use of the Vacuum Casting method in the process of making the model, individual elements and structures will have a separate production process, and the final one will be flooding all structures with each other. The existing methodology will have to be separate depending on the part produced. For the elements that do not require imitation of soft tissue, the only task of which is to image the distribution with respect to pathological changes (e.g. blood vessels), it is possible to directly create a structure model from the digital model. In the case of the elements manufactured using the method of Vacuum Casting, it is necessary to create a form based on the digital record of the 3D model of the organ with structures and changes. It is an output element the task of which is to connect all related structures into one physical model. It is important to precisely and precisely design the form so that precise positioning of the structures with each

Table 3. Selected parameters for the material Dragon Skin 10 Very Fast

\begin{tabular}{|c|c|}
\hline & Dragon Skin ${ }^{T M} 10$ VERY FAST \\
\hline Product Type & Silicone Rubber - Platinum Cure \\
\hline Mixed Viscosity & $23,000 \mathrm{cps}$ \\
\hline Mix Ratio By Volume & $1 \mathrm{~A}: 1 \mathrm{~B}$ \\
\hline Mix Ratio By Weight & $1 \mathrm{~A}: 1 \mathrm{~B}$ \\
\hline Pot Life & 4 minutes \\
\hline Cure Time & 30 minutes \\
\hline Shore A Hardness & 10 \\
\hline Specific Gravity & $1.07 \mathrm{~g} / \mathrm{cc}$ \\
\hline Specific Volume & $25.8 \mathrm{cu}$. in./lb. \\
\hline Color & Translucent \\
\hline Die B Tear Strength & $102 \mathrm{pli}$ \\
\hline Tensile Strength & 475 psi \\
\hline Elongation $₫$ Break & $1,000 \%$ \\
\hline $100 \%$ Modulus & 22 psi \\
\hline Shrinkage & $<.001$ in. / in. \\
\hline Useful Temperature (min) & $-65^{\circ} \mathrm{F}$ \\
\hline Useful Temperature (max) & $450^{\circ} \mathrm{F}$ \\
\hline
\end{tabular}




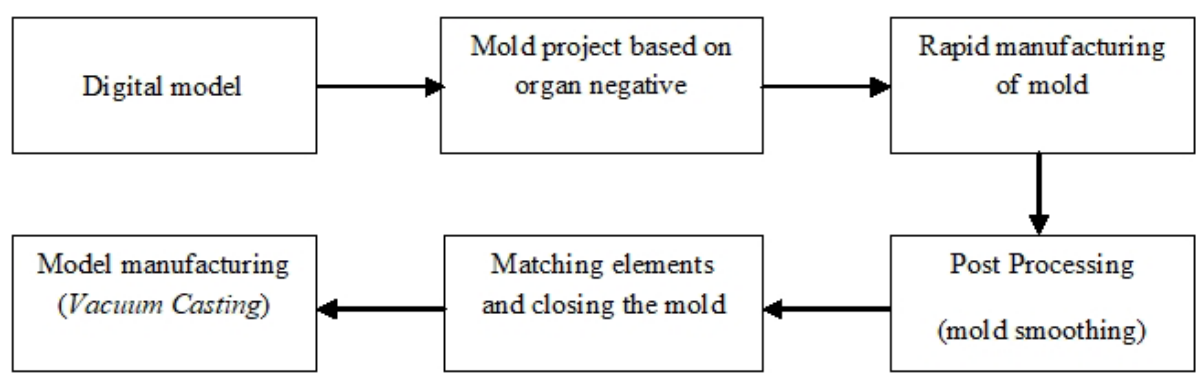

Fig. 8. Methodology of manufacturing combined models

other is possible. Changing the distribution of all elements may affect the credibility of the model. The methodology of this part of the process is shown in Figure 8. The digital model of the organ with all structures is the basis for designing the casting mold. The form contains a negative of the final model. The design process takes place in a CAD environment or software that allows working on a triangle grid. The mold design must be optimized; the process cannot be long-lasting to prevent extending the production process and increasing the material consumption, which translates into an increase in the price of the final model. In addition, the form must contain all the elements necessary for the correct casting process. After the mold has been made, it is necessary to smoothen the inner surface in order to cover the stepped effect, distorting the actual appearance of the form and disturbing the view of the structure inside the transparent model. The constituent elements should be placed in a mold so that they do not move during the material introduction. The sealed mold can be subjected to a vacuum casting process. The obtained model becomes a multi-material object that can be used to perform a simulated operation.

\section{CONCLUSION}

The 3D Printing technology, by making the model layer-by-layer based on the digital model, allows obtaining the shapes that are impossible to achieve using other technologies. That is why such an intensive development of methods of rapid manufacturing has a significant impact on medicine and gradually implemented new applications of printed products.

The selection of materials dedicated to low-cost 3D printing methods was associated with a subjective assessment, which was obtained on several levels. Due to the specific nature of the source models (living human organs), it was difficult to fully obtain the necessary information about the material that is supposed to imitate the soft tissues of the kidney. The basis for determining the parameters was to refer to the animal model in the form of pig kidney. The tests performed on the organ were used for further selection of materials. At the same time, the information was collected during consultation with a specialist surgeon, who determined certain desirable parameters (price, transparency) and helped to determine the others (by performed simulated operation on an example of a kidney model). On the basis of the knowledge of the required features, it was possible to narrow the group of materials to one method of rapid manufacturing - vacuum casting. Further selection of materials was associated with a series of studies based on surgical suturing, observations and surveys conducted among a group of students. The presented proposal to create, at a low cost, a multi-material anatomical model is aimed at creating a pre-operative personalized for a particular patient, which will be used for detailed preparation for surgery. In countries like Poland, the cost of the model cannot be significant, especially in the cases of low and medium complexity. Excessively high a cost would make it impossible to introduce such models to the standard of preoperative surgeon preparation.

In addition, individual anatomical models have an educational nature. They enable to become acquainted with the actual case of the patient, transferred reliably by using additive manufacturing technologies on the anatomical model. Expanding the knowledge of students about such cases will allow to better acquisition of the knowledge related to everyday and rare cases of lesions. During the survey, $100 \%$ of participants expressed their willingness to work on the models enabling surgical sewing and conducting simulated operations. 
Further research will cover other groups of materials, in particular the method of permanently joining them together, as well as searching for the materials enabling the character of the pathological change, especially in the kidneys. At the same time, further research will be carried out to create a more accurate method of material evaluation and to shorten the production time of the multi-material models.

\section{Acknowledgements}

The studies were realized with a support from statutory activity financed by Polish Ministry of Science and Higher Education (02/23/DSMK/8722).

\section{REFERENCES}

1. Banaszewski J., et al., 3D printed models in mandibular reconstruction with bony free flaps, Journal of Materials Science: Materials in Medicine, 29, 2018, 23, https://doi.org/10.1007/s10856-018-6029-5

2. European Association of Urology, Surgeons develop personalised $3 \mathrm{D}$ printed kidney to simulate surgery prior to cancer operation [online], [access: July 2017], <http://www.alphagalileo.org/ViewItem.aspx?ItemId $=140891 \&$ CultureCode $=$ en $>$

3. Faulkner-Jones, et. al., Bioprinting of human pluripotent stem cells and their directed differentiation into hepatocyte-like cells for the generation of mini-livers in 3D, Biofabrication, 7(4), 2015.
4. Gibson, et. al., The use of Rapid Prototyping to assist medical applications. Rapid Prototyping Journal, 12(1), 2006, 53-58.

5. Górski F., et al., Mechanical properties of parts of medical components produced using additive manufacturing technologies., Advances in Science and Technology Research Journal, 11(2), 2017, 166-171, DOI: 10.12913/22998624/70997

6. John T. Milliken, 3D printed kidney models change the way surgeons prepare for surgery [online], [acsess: July 2017], https://renal.wustl.edu/3d-printed-kidney-models-change-way-surgeons-prepare-surgery/

7. Nadav Noor, et. al., 3D printing of personalized thick and perfusable cardiac patches and hearts, Advanced Science 2019.

8. Radosh A., et al., Prototyping of cosmetic prosthesis of upper limb using additive manufacturing technologies. Advances in Science and Technology Research Journal, 11(3), 2017, 102-108, DOI: 10.12913/22998624/70995

9. Silberstein L. et. al., Physical models of renal malignancies, using standard cross-sectional imaging and 3-dimensional printers: A pilot study. Urology, 84(2), 2014, 268-273.

10. Wierzbicka N., et al., Prototyping of individual ankle orthosis using additive manufacturing technologies. Advances in Science and Technology Research Journal, 11(3), 2017, 283-288, DOI: $10.12913 / 22998624 / 76070$

11. Zhou G. et. al., In vitro regeneration of patient-specific ear-shaped cartilage and its first clinical application for auricular reconstruction, EBioMedicine 28, 2018, 287-302. 